\title{
Is it severe asthma or asthma with severe comorbidities?
}

Luisa Brussino'

Paolo Solidoro ${ }^{2}$

Giovanni Rolla'

'Allergy and Clinical Immunology, University of Torino and Mauriziano Hospital, ${ }^{2} \mathrm{SC}$ Pneumologia U,AOU Città della Salute e della Scienza, Torino, Italy
Correspondence: Giovanni Rolla Allergy and Clinical Immunology, University of Torino and Mauriziano Hospital, Largo Turati, 62, I0I 28 Torino, Italy

Tel +39 0l I 5082926

Email grolla@mauriziano.it
This article was published in the following Dove Press journal: Journal of Asthma and Allergy

\begin{abstract}
Severe asthma is defined as asthma that requires treatment with high-dose inhaled corticosteroids (ICSs) plus a second controller and/or systemic corticosteroids to prevent it from becoming uncontrolled or that remains uncontrolled despite this therapy. This definition has limitations: 1) it does not define any biological characteristic that distinguishes severe asthma from asthma in general and 2) it relies on the clinical interpretation of asthma symptoms that are not specific. Actually, wheezing, dyspnea, cough and chest tightness may be caused by the comorbidities (such as rhinosinusitis, obesity and vocal cord dysfunction [VCD]) which are associated with asthma. In clinical practice, clinicians are often prone to diagnose uncontrolled asthma and increase doses of ICSs without considering the comorbidities, resulting in poor control of symptoms. This commentary wishes the clinicians to focus on the comorbidities of asthma, particularly in patients with severe asthma, because the correct diagnosis of these comorbidities implies specific treatments that lead to a better asthma control.
\end{abstract}

Keywords: severe asthma, chronic rhinosinusitis, obesity, vocal cord dysfunction, dupilumab

The recent increase in therapeutic options for the treatment of severe asthma ${ }^{1}$ has increased the importance of diagnosis and asthma phenotyping. According to International European Respiratory Society (ERS)/American Thoracic Society (ATS) task force, severe asthma is defined as asthma that requires treatment with high-dose ICSs plus a second controller and/or systemic corticosteroids to prevent it from becoming uncontrolled or that remains uncontrolled despite this therapy. ${ }^{2}$ The criteria for uncontrolled asthma include: poor symptom control, frequent severe exacerbations or one serious exacerbation that requires hospitalization, intensive care unit (ICU) stay or mechanical ventilation in the previous year or airflow limitation. This definition has limitations: it does not define any biological characteristic that distinguishes severe asthma from asthma in general and it relies on the clinical interpretation of symptoms that should be attributed to asthma. However, asthma has no characteristic symptoms because wheezing, dyspnea, cough and chest tightness are the symptoms that are not specific, as these are caused by the comorbidities associated with asthma. Here, we use the term asthma comorbidity to refer to a condition that influences asthma severity, management or diagnosis, with consequent misattribution of shared symptoms. In pediatric patients, an association of asthma with cystic fibrosis (CF) may occur, by coincidence to the high prevalence of asthma in children, the so-called CF asthma. ${ }^{3}$ The patient with CF is reported as having concomitant asthma if, in the treating physician's opinion, asthma contributes significantly to the patient's lung disease. The diagnosis of 
asthma is suggested by episodes of acute airway obstruction reversed by bronchodilators, a strong family history of asthma and/or evidence of atopy or laboratory evidence of allergy (ie, prick tests positive for common inhalant allergens). In the elderly, it is not uncommon to evaluate patients with persistent airflow limitation and smoking history, with some features usually associated with asthma, such as eosinophilic airway inflammation, the so-called asthma COPD overlap syndrome (ACOS), as defined by recent consensus criteria. ${ }^{4}$ Clinicians should enquire about smoking habit of their difficult-to-treat asthma patients, as smoking is associated with reduced expression of histone deacetylase (HDAC2). HDAC2 appears to mediate the action of steroids to switch off activated inflammatory genes, but in smokers with asthma, HDAC2 activity and expression are reduced by oxidative stress through activation of phosphoinositide 3-kinase $\delta .^{5}$ We will consider in more detail some comorbidities which, based upon the authors' experience, have a major impact on the management of asthmatic patients. In the clinical scenario of a patient who had previously received the diagnosis of asthma, whenever cough, dyspnea and wheezing are due to asthma comorbidities, such as rhinosinusitis, obesity and VCD, clinicians are prone to diagnose uncontrolled asthma and increase doses of ICSs, resulting in poor control of symptoms. This condition fulfills the criteria of severe asthma, and the correct diagnosis should be asthma with severe comorbidities.

The comorbidities may result in misdiagnosis, misinterpretation of symptoms, aggravation of one or more diseases. In contrast, the recognition of comorbidities facilitates more appropriate therapy or reduction of potentially risky therapies, such as systemic corticosteroids.

Specific treatments of comorbidities have been shown to improve asthma control. According to Ragab et al, ${ }^{6}$ both medical and surgical treatments of chronic rhinosinusitis (CRS) were associated with subjective and objective improvements in asthma. Some new biological therapies targeting IL5 and IL4/IL13 may improve nasal polyps score and decrease asthma exacerbation rate. ${ }^{1,7,8}$ The proportion of patients with symptoms of CRS has been reported to be as high as $74 \%$ in patients with severe steroid-dependent asthma, who showed a severe sino-nasal involvement as evaluated by computed tomography (CT) scan imaging. ${ }^{9}$ Clinicians should consider the diagnosis of CRS based upon the presence of at least two of the four cardinal signs/symptoms (anterior and/or posterior nasal mucopurulent drainage, nasal obstruction/ nasal blockage/congestion, facial pain, pressure and/or fullness and reduction or loss of sense of smell) in combination with objective evidence of mucosal inflammation. ${ }^{10}$ Objective evidence of mucosal inflammation requires demonstration of one or more of the following findings, using nasal endoscopy and/or CT imaging: purulent mucus or edema in the middle meatus or ethmoid regions, polyps in the nasal cavity or the middle meatus, radiographic imaging demonstrating mucosal thickening or partial or complete opacification of the paranasal sinuses. In obese patients, the risk of misdiagnosing asthma and/or its severity is particularly high. Among 91 subjects (mean body mass index [BMI], $38 \mathrm{~kg} / \mathrm{m}^{2}$ ) with a diagnosis of asthma, taking a mean beclomethasoneequivalent dose of $1,273 \mathrm{mg} /$ day, $36.3 \%$ had no bronchial hyperresponsiveness (possible misclassification of asthma diagnosis). ${ }^{11}$ Therefore, clinicians should not accept asthma diagnosis based on symptoms alone particularly in obese subjects. The objective measurements of variable airflow obstruction or bronchial hyperresponsiveness are important to confirm the diagnosis of asthma in obese patients. If obese patients who are receiving asthma treatment are still reporting dyspnea and exercise intolerance, it is advised to check lung function and, if normal, to step down asthma therapy and recheck respiratory function. Otherwise, a misdiagnosis may lead to inappropriate treatment, with an increased risk of side effects and increased costs. ${ }^{12}$ On the other hand, bariatric surgery not only improved small airway function, which could explain the improvement of asthma control and quality of life, but also induced a decrease in systemic and bronchial inflammation in morbidly obese patients with asthma. ${ }^{13}$

Prevalence of dysfunctional breathing, evaluated by Nijmegen questionnaire, in patients treated for asthma in primary care has been estimated to be $35 \%$ among women and $20 \%$ among men, ${ }^{14}$ and reports of VCD mimicking severe asthma are not rare. ${ }^{15,16} \mathrm{VCD}$ is defined by the complete or partial adduction of the vocal folds with inspiration and/or expiration. This process seems to occur in response to irritation of the larynx or hypopharynx or secondary to emotional or physical stress. ${ }^{17}$ The symptoms of VCD include high-pitched wheezing, usually more prominent with inspiration, hoarseness, dysphonia and cough. ${ }^{18}$ Symptoms are often episodic with rapid resolution, with or without therapy. Patients may be misdiagnosed with asthma when VCD is responsible for their symptoms or have VCD and asthma simultaneously. The use of the flow volume loop may be helpful in recognizing VCD. The inspiratory loop typically exhibits decreased flow rate with variability in flow, resulting in a wavy, flattened curve instead of the typical smooth, oval appearance. A speech therapist or speech pathologist, knowledgeable about VCD, can be very helpful in treating this disorder. ${ }^{19}$ Over half of the patients treated for asthma in the community who have 
symptoms suggestive of dysfunctional breathing showed a clinically relevant improvement in quality of life following a brief physiotherapy intervention, and the improvement was maintained to be over $25 \% 6$ months after the intervention. ${ }^{20}$ In conclusion, we wish the clinicians to focus on the comorbidities of asthma, particularly in patients with severe asthma, because the correct diagnosis of these comorbidities implies specific treatments that lead to a better asthma control.

\section{Disclosure}

The authors report no conflicts of interest in this work.

\section{References}

1. Menzella F, Galeone C, Bertolini F, et al. Innovative treatments for severe refractory asthma: how to choose the right option for the right patient? J Asthma All. 2017;10:237-247.

2. Chung KF, Wenzel SE, Brozek JL, et al. International ERS/ATS guidelines on definition, evaluation and treatment of severe asthma. Eur Respir J. 2014;43(2):343-373.

3. Balfour-Lynn IM, Elborn JS. "CF asthma": what is it and what do we do about it? Thorax. 2002;57:742-748.

4. Bonten TN, Kasteleyn MJ, De Mutsert R, et al. Defining asthma-COPD overlap syndrome: a population-based study. Eur Respir J. 2017;49(5): 1602008 .

5. Barnes PJ. Corticosteroid resistance in patients with asthma and chronic obstructive pulmonary disease. J Allergy Clin Immunol. 2013;131(3):636-645.

6. Ragab S, Scadding GK, Lund VJ, et al. Treatment of chronic rhinosinusitis and its effects on asthma. Eur Respir J. 2006;28(1):68-74.

7. Gevaert P, Van Bruaene N, Cattaert T, et al. Mepolizumab, a humanized anti-IL-5 $\mathrm{mAb}$, as a treatment option for severe nasal polyposis. J Allergy Clin Immunol. 2011;128(5):989-995.
8. Bachert C, Mannent L, Naclerio RM, et al. Effect of subcutaneous dupilumab on nasal polyp burden in patients with chronic sinusitis and nasal polyposis: a randomized clinical trial. JAMA. 2016;315(5):469-479.

9. Bresciani M, Paradis L, Des Roches A, et al. Rhinosinusitis in severe asthma. J Allergy Clin Immunol. 2001;107(1):73-80.

10. Rosenfeld RM, Piccirillo JF, Chandrasekhar SS, et al. Clinical practice guideline (update): adult sinusitis. Otolaryngol Head Neck Surg. 2015;152(2 suppl):S1-S39.

11. Scott S, Currie J, Albert P, Calverley P, Wilding JPH. Risk of misdiagnosis, health-related quality of life, and BMI in patients who are overweight with doctor-diagnosed asthma. Chest. 2012;141(3):616-624.

12. Van Huisstede A, Castro Cabezas M, van de Geijn GJ, et al. Underdiagnosis and overdiagnosis of asthma in the morbidly obese. Respir Med. 2013;107(9):1356-1364.

13. van Huisstede A, Rudolphus A, Castro Cabezas M, et al. Effect of bariatric surgery on asthma control, lung function and bronchial and systemic inflammation in morbidly obese subjects with asthma. Thorax. 2015;70(7):659-667.

14. Thomas M, McKinley RK, Freeman E, et al. Prevalence of dysfunctional breathing in patients treated for asthma in primary care: cross sectional survey. 1. BMJ. 2001;322(7294):1098-1100.

15. Wolfe JM, Meth BM. Vocal cord dysfunction mimicking a severe asthma attack. J Emerg Med. 1999;17(1):39-41.

16. Balkissoon R, Kenn K. Asthma: vocal cord dysfunction (VCD) and other dysfunctional breathing disorders. Semin Respir Crit Care Med. 2012;33(6):595-605.

17. Bucca CB, Bugiani M, Culla B, et al. Chronic cough and irritable larynx. J Allergy Clin Immunol. 2011;127:412-419.

18. Fretzayas A, Moustaki M, Loukou I, et al. Differentiating vocal cord dysfunction from asthma. J Asthma Allergy. 2017;10:277-283.

19. Watson MA, King CS, Holley AB, Greenburg DL, Mikita JA. Clinical and lung-function variables associated with vocal cord dysfunction. Respir Care. 2009;54(4):467-473.

20. Thomas M, McKinley RK, Freeman E, et al. Breathing retraining for dysfunctional breathing in asthma: a randomised controlled trial. Thorax. 2003;58(2):110-115.
Journal of Asthma and Allergy

\section{Publish your work in this journal}

The Journal of Asthma and Allergy is an international, peer-reviewed open access journal publishing original research, reports, editorials and commentaries on the following topics: Asthma; Pulmonary physiology; Asthma related clinical health; Clinical immunology and the immunological basis of disease; Pharmacological interventions and

\section{Dovepress}

new therapies. This journal is included in PubMed. The manuscript management system is completely online and includes a very quick and fair peer-review system, which is all easy to use. Visit http://www. dovepress.com/testimonials.php to read real quotes from published authors. 